\title{
Utilização da ultrassonografia para guiar punção venosa central em hospitais de Manaus
}

\author{
Use of ultrasonography to guide central venous catheterization in Manaus hospitals
}

Uso de la ecografía para guiar la cateterización venosa central en hospitales de Manaus

Anfremon D'Amazonas Monteiro Neto ${ }^{1}$, Renata Motoki Amorim Pereira ${ }^{1}$, Rosane Dias da Rosa ${ }^{1}$, Fernando Luiz Westphal ${ }^{1 *}$, Juscimar Carneiro Nunes ${ }^{1}$.

\section{RESUMO}

Objetivo: Identificar a frequência de utilização da ultrassonografia para guiar punções venosas centrais em hospitais terciários em Manaus/Amazonas. Métodos: Estudo observacional, transversal e descritivo realizado entre novembro/2017 e agosto/2018. Foi aplicado um questionário eletrônico aos médicos que puncionam tais acessos em três hospitais públicos e um privado. Avaliou-se a frequência da utilização de ultrassom e principais empecilhos para sua adoção. Para a análise dos dados foi usado o programa Epi Info, com nível de significância de 5\%. Resultados: Foram entrevistados 200 médicos, sendo $86,5 \%$ de instituições públicas e $46 \%$ de especialistas. Apesar da maioria (74\%) já ter usado o ultrassom anteriormente, a taxa de uso foi de $43,9 \%$. A principal dificuldade foi a indisponibilidade imediata do aparelho $(68,2 \%)$. Entre os $26 \%$ que nunca utilizaram o método, a falta de treinamento $(69,2 \%)$ foi a barreira preponderante. Dificuldades anatômicas $(80 \%)$, obesidade $(70 \%)$ e distúrbios de coagulação $(67 \%)$ foram os principais motivadores ao uso da ultrassonografia. Participantes com idade superior a 50 anos utilizavam menos a ultrassonografia, porém sem significância estatística $(p=0,062)$. Conclusão: Apesar dos conhecidos benefícios, a ultrassonografia não é utilizada rotineiramente nos hospitais da cidade. Treinamento adequado e facilitação de acesso aos equipamentos parecem ser a forma de melhorar este cenário.

Palavras-chave: Cateterismo venoso central, Ultrassom, Ultrassonografia de intervenção.

\section{ABSTRACT}

Objective: To identify the use rate of ultrasound-guided technique for central venous catheter placement in tertiary hospitals in Manaus/Amazonas. Methods: Observational, cross-sectional and descriptive study carried out between November/2017 and August/2018. An electronic questionnaire was given to doctors who insert such access in three public and one private hospital. The frequency of ultrasound guidance and the main obstacles for its use were evaluated. For data analysis, the Epi Info program was used, with a 5\% significance level. Results: 200 doctors were interviewed, $86.5 \%$ from public institutions and $46 \%$ were specialists. Despite the majority $(74 \%)$ stating prior ultrasound use, the usage rate was $43.9 \%$. The main difficulty was the lack of immediate availability of the device $(68.2 \%)$. Among the $26 \%$ who never used the method, lack of training $(69.2 \%)$ was the main barrier. Anatomical difficulties (80\%), obesity $(70 \%)$ and coagulation disorders $(67 \%)$ were the leading motivators for the ultrasound use. Participants over the age of 50 reported less ultrasound use, but without statistical significance $(p=0.062)$. Conclusion: Despite the known benefits, ultrasonography is not routinely used in the city hospitals. Adequate training and easier access to devices seem to be the way of improving this scenario.

Key words: Central venous catheterization, Ultrasonics, Interventional ultrasonography.

\section{RESUMEN}

Objetivo: Identificar la frecuencia de utilización de la ultrasonografía para orientar los accesos venosos centrales en hospitales terciarios de Manaus/Amazonas. Métodos: Estudio observacional, transversal y 1 Universidade Federal do Amazonas - Programa de Pós-Graduação em Cirurgia (PPGRACI), Manaus -
Amazonas. *E-mail: f.l.westphal@gmail.com

Fundação de Amparo à Pesquisa do estado do Amazonas (FAPEAM) por meio do Programa de Apoio à Publicações Científicas (PAPAC). Edital 005/2019.

SUBMETIDO EM: 1/2021 ｜ＡCEITO EM: 1/2021 ｜ PUBLICADO EM: 2/2021 
descriptivo realizado entre noviembre/2017 y agosto/2018. Se aplicó un cuestionario electrónico a los médicos que hacen dichos accesos en tres hospitales públicos y uno privado. Se evaluó la frecuencia de uso y principales obstáculos para la adopción del ultrasonido. Para el análisis de datos se utilizó el programa Epi Info, con nivel de significancia de 5\%. Resultados: Se entrevistaron 200 médicos, 86,5\% de instituciones públicas y $46 \%$ de especialistas. Aunque la mayoría (74\%) había utilizado el ultrasonido anteriormente, la frecuencia fue de 43,9\%. La principal dificultad fue la falta de disponibilidad inmediata del dispositivo (68,2\%). Entre los $26 \%$ que nunca usaran, falta de formación $(69,2 \%)$ fue la principal barrera. Dificultades anatómicas $(80 \%)$, obesidad (70\%) y trastornos de la coagulación $(67 \%)$ fueron los principales motivadores para su uso. Los participantes mayores de 50 años utilizaron menos la ecografía, pero sin significación estadística $(p=0,062)$. Conclusión: Mismo con los beneficios conocidos, la ecografía no se utiliza rutinariamente en los hospitales de la ciudad. Una formación adecuada y un acceso más fácil a los dispositivos pueden mejorar este escenario.

Palabras clave: Cateterismo venoso central, Ultrasonido, Ultrasonografía intervencional.

\section{INTRODUÇÃO}

A punção venosa central é um procedimento habitual na medicina moderna e tem várias aplicações no âmbito terapêutico e diagnóstico. Apesar de ser executado de forma rotineira nas unidades de saúde, é um procedimento associado a diversas complicações com frequência não desprezível, entre as quais é possível citar: falha em estabelecer o acesso venoso, infecção associada ao cateter, punção arterial, hematoma subcutâneo, pneumotórax, hemotórax, estenose ou trombose venosa, e, em casos raros, parada cardíaca súbita (YOUNG MP, YOU TH, 2020; BANNON MP, 2011).

Os relatos iniciais do uso da ultrassonografia (USG) para guiar esses acessos são da década de oitenta, inicialmente de forma indireta com a marcação do local a ser puncionado após a identificação da veia subclávia ou jugular interna através do Doppler (PETERS JL, et al., 1982; LEGLER D e NUGENT M, 1984) e posteriormente pela técnica de punção sob visualização direta (YONEI A, et al., 1986). Nos anos seguintes, várias pesquisas mostraram a eficácia e a segurança deste método em comparação com a técnica tradicional que utiliza pontos anatômicos na pele do paciente como referência para direcionar a agulha, a chamada "Técnica Anatômica". A ultrassonografia (USG) proporciona maior taxa de acerto na primeira punção, maior índice de sucesso e menor taxa de complicações, sendo seu uso sistemático recomendado para guiar as punções tanto de forma eletiva quanto nos cenários de urgência (BRASS P, et al., 2015; LAMPERTI M, et al., 2012; NATIONAL INSTITUTE FOR CLINICAL EXCELLENCE, 2002; TROIANOS CA, et al., 2012; FRANCOSADUD R, et al., 2019).

Os benefícios da USG no acesso venoso central também se traduzem no âmbito econômico, Noritomi DT, et al. (2016) realizaram uma simulação teórica comparando a ultrassonografia em tempo real versus a técnica tradicional e, considerando a incorporação da tecnologia e a diminuição do custo pela redução das complicações, houve uma economia de $\mathrm{R} \$ 74,33$ por acesso venoso central inserido. A razão de custoefetividade incremental indicou uma economia de $\mathrm{R} \$ 2.494,34$ por caso de pneumotórax evitado ao se usar a USG em comparação à técnica anatômica, revelando uma redução hipotética de gastos através da implementação da tecnologia pelo Sistema Único de Saúde.

A inserção do cateter sob visualização direta é especialmente interessante em determinados grupos, como nos obesos mórbidos, em pacientes com variações anatômicas e na população pediátrica (KASATKIN AA, et al. 2018; SAUGEL B, et al., 2017; LAU CS e CHAMBERLAIN RS, 2016). O acesso venoso central em crianças, principalmente em lactentes, é dificultado pelo tamanho de veia reduzido, menor cooperação do paciente e variações anatômicas mais frequentes. A USG aumenta o êxito na primeira tentativa, reduz o número de punções necessárias para obtenção do acesso além de reduzir o número de punções arteriais inadvertidas e complicações nesse público, em especial quando o operador possui menos experiência (OULEGO-ERROZ I, et al., 2018).

Em diversos lugares do mundo, a despeito das evidências científicas comprovando sua eficácia e benefícios, a USG para guiar a implantação de cateteres venosos centrais não é usada rotineiramente, falta de treinamento e ausência de aparelhos nos locais de trabalho são causas relatadas com frequência para a 
adoção parcial desta técnica (GREBENIK CR, et al., 2004). Todavia, em países onde há aparelhos e treinamento, os índices de adesão também permanecem relativamente baixos, sendo a ideia de que o ultrassom não é necessário para guiar as punções e a indisponibilidade imediata do equipamento, os principais responsáveis por tais taxas (MAIZEL J, et al., 2016).

Na Bélgica, França e Suíça, a frequência de uso do ultrassom é de apenas $54 \%$ mesmo havendo aparelho de USG em todas as unidades (ZIELESKIEWICZ L, et al., 2015). Na Holanda, cerca de um terço dos intensivistas e anestesiologistas ainda se apoia na técnica anatômica para a inserção dos cateteres. As barreiras ao uso incluem a falsa percepção de que a USG aumenta o tempo de procedimento, receio de perder a habilidade de se orientar por marcos anatômicos, indisponibilidade do equipamento e ausência de benefício percebido com a técnica ultrassonográfica (SCHOLTEN HJ, et al., 2019).

Dessa forma, a falta de adesão a este método comprovadamente mais seguro e eficaz enfrenta problemas estruturais e de políticas de saúde, como falta de equipamentos e de treinamento dos profissionais, que levam a falência em reconhecer a importância da utilização da tecnologia, desconhecimento de seus benefícios, ideias equivocadas sobre o tempo de procedimento e resistência a mudanças (GREBENIK CR, et al., 2004; SCHOLTEN HJ, et al., 2019). Como não foram encontrados estudos sobre o tema em questão na região amazônica, o comportamento dos médicos na capital do Amazonas em relação ao uso da USG para guiar a implantação de cateteres venosos centrais é desconhecido e um melhor entendimento dessa realidade local possibilitaria a implementação de protocolos a fim de melhorar o uso da tecnologia e aumentar a segurança dos pacientes submetidos ao procedimento.

A escassez destas informações suscitou a realização deste estudo que busca verificar a frequência de uso do ultrassom para guiar tais acessos e determinar os fatores que podem facilitar ou dificultar seu uso.

\section{MÉTODOS}

Estudo observacional descritivo transversal executado entre novembro de 2017 a agosto de 2018, por meio de formulário eletrônico de 13 a 16 perguntas (variando de acordo com as respostas prévias) aplicado aos participantes. O questionário de Maizel J, et al. (2016) foi utilizado como base tendo sido traduzido e adaptado de forma livre pelos pesquisadores deste trabalho para melhor adequar-se às circunstâncias locais com a devida anuência do autor. A plataforma eletrônica para elaboração, aplicação do inquérito e coleta dos dados foi a KoboToolBox® que permitiu que a coleta fosse realizada por meio de tablets. A pesquisa foi submetida ao Comitê de Ética em Pesquisa (CEP) e obteve aprovação com parecer consubstanciado número 2.302.741 e CAAE 77153917.7.0000.5020.

O questionário foi aplicado a um grupo piloto com cinco participantes e não foram encontradas dificuldades para responder às perguntas. A amostra foi composta por médicos que realizam a inserção de cateter venoso central em sua rotina diária, dentre os quais, clínicos (incluindo subespecialidades), intensivistas, emergencistas, anestesiologistas, cirurgiões e residentes das áreas citadas. Participaram da pesquisa os profissionais de três hospitais públicos de Manaus, um hospital universitário de administração federal (Hospital 1), uma unidade hospitalar da rede de urgência e emergência estadual (Hospital 2) e uma fundação hospitalar estadual (Hospital 3), e de um hospital da rede privada (Hospital 4). Foram priorizadas as unidades hospitalares no mínimo terciárias, por serem locais onde os procedimentos percutâneos estudados são realizados com maior frequência.

Pela ausência de estimativa do número de médicos que realizam o procedimento de acesso venoso central em sua prática habitual e pela duplicidade de médicos trabalhando em mais de um dos hospitais estudados, optou-se por uma amostra de conveniência. Dos 794 médicos empregados nas instituições estudadas, foram entrevistados 200 profissionais. Aqueles que já tinham respondido o formulário no estudo piloto e aqueles que nunca realizaram uma punção de veia central foram excluídos do estudo. Os entrevistadores aplicaram o questionário nas respectivas unidades de trabalho a todos aqueles que atenderam aos critérios de inclusão e concordaram em assinar o Termo de Consentimento Livre e Esclarecido (TCLE) após leitura. A abordagem dos entrevistadores foi física, direta e aleatória, variando os cenários dentro de cada hospital: enfermaria, pronto socorro, Unidade de Terapia Intensiva (UTI) e centro cirúrgico. 
$\mathrm{Na}$ análise dos dados foi aplicado o teste Qui-quadrado de Pearson e o teste de Fisher por meio do programa Epi Info versão 7.2.2 para Windows. O nível de significância fixado na aplicação dos testes estatísticos foi de $5 \%$.

\section{RESULTADOS}

Foram entrevistados 200 médicos, sendo 173 (86,5\%) de instituições públicas e 27 (13,5\%) da rede privada, no período de novembro de 2017 a agosto de 2018. As coletas se distribuíram da seguinte forma: 61 $(30,5 \%)$ profissionais do Hospital 3, $59(29,5 \%)$ do Hospital 2, $53(26,5 \%)$ do Hospital 1 e 27 (13,5\%) do Hospital 4. O hospital privado, o Hospital 4, tem menor porte quando comparado as outras instituições, por isso a coleta nesse estabelecimento foi proporcionalmente inferior.

Dos profissionais que responderam ao questionário, 92 (46\%) eram especialistas, 77 (38,5\%) residentes de acesso direto ou área básica, 16 (8\%) residentes de especialidades cirúrgicas ou clínicas e 15 (7,5\%) médicos sem especialidade e não residentes. Ao agrupar os dois grupos de residentes, é possível observar uma distribuição homogênea entre especialistas 92 (46\%) e residentes $93(46,5 \%)$, dessa forma, foi usado este conjunto para a análise estatística dos dados (Tabela 1).

Tabela 1 - Distribuição dos profissionais de acordo com a especialização, em hospitais terciários da cidade de Manaus-AM, novembro de 2017 a agosto de 2018 ( $n=200)$.

\begin{tabular}{lcc}
\hline Área & $\mathbf{n}$ & $\%$ \\
\hline Especialista & 92 & 46 \\
$\begin{array}{l}\text { Residente de Área Básica (Clínica Médica, Cirúrgica, } \\
\text { Pediatria, etc.) ou Acesso Direto (Anestesiologia, }\end{array}$ & 77 & 38,5 \\
$\begin{array}{l}\text { Dermatologia, Infectologia) } \\
\text { Residente de área de atuação (Medicina Intensiva, }\end{array}$ & & \\
Gastroenterologia, Urologia, Cirurgia Vascular) & 16 & 8 \\
Não residente e não especialista. & 15 & 7,5 \\
\hline
\end{tabular}

Fonte: Monteiro Neto AD, et al., 2021.

A distribuição dos entrevistados conforme os locais onde costumam puncionar os acessos mostrou uma prevalência da opção hospital público $(61,5 \%)$. Esta pergunta permitia que o entrevistado assinalasse múltiplas respostas, dessa forma, um médico que trabalhe no turno da manhã em uma UTI de um hospital particular e à noite em um pronto socorro de um hospital universitário público, assinalaria todas as quatro alternativas. É possível observar que a quantidade de médicos que dizem realizar as punções na rede privada $(35,5 \%)$ é bem superior ao número de profissionais que responderam à pesquisa no hospital privado (14,5\%), demonstrando que muitos dos entrevistados na rede pública também exercem sua atividade laboral na rede particular. Este ato de percorrer ambas as esferas, pública e privada, aparenta ser uma particularidade da comunidade médica local (Figura 1).

Figura 1 - Distribuição dos médicos de acordo com os locais mais frequentes para a realização de punções venosas centrais $(n=200)$.

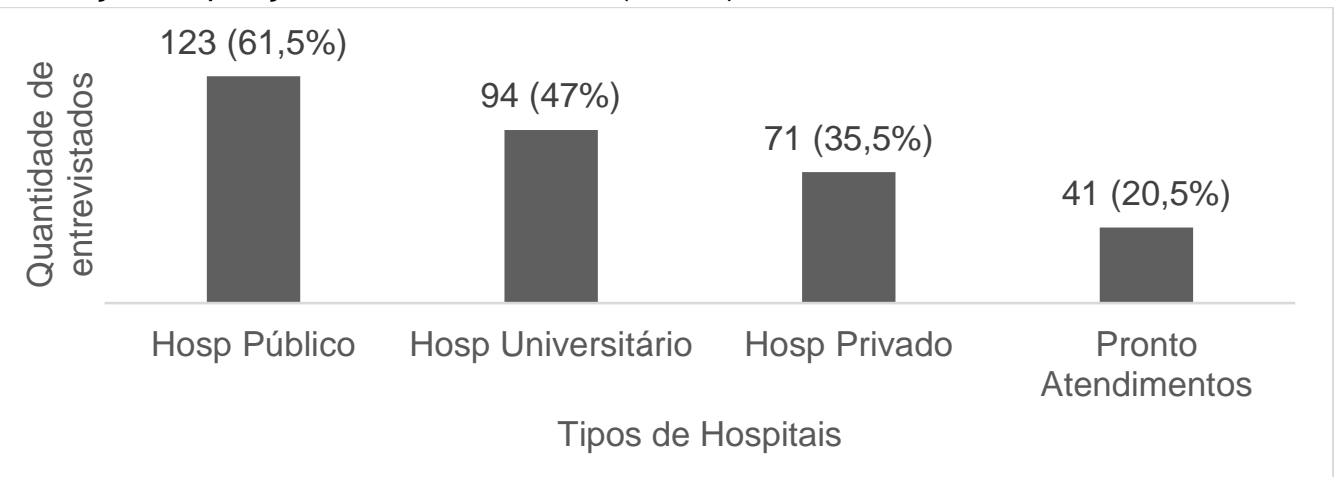

Fonte: Monteiro Neto AD, et al., 2021. 
A maioria dos entrevistados, 148 (74\%), referiu já ter feito uso da USG para guiar acessos venosos centrais. Dentre as barreiras encontradas para a utilização da tecnologia, 123 (61,5\%) médicos apontaram a indisponibilidade imediata do equipamento, $49(24,5 \%)$ citaram inexistência do aparelho no local de trabalho e $69(34,5 \%)$ selecionaram falta de treinamento como motivo.

No grupo que já utilizou ao menos uma vez a punção guiada pelo ultrassom, $83(56,1 \%)$ referiram quase nunca usar a tecnologia como guia. Entre as razões para a não utilização da USG, 101 (68,2\%) participantes alegaram a indisponibilidade imediata do aparelho, 40 (27\%) a ausência do equipamento na unidade e 33 $(22,3 \%)$ a falta de treinamento. Esta pergunta permitia assinalar mais de uma opção. Houve uma concordância de respostas entre os residentes e os especialistas. Já no grupo dos não residentes/ especialistas houve uma tendência a disparidade em certas respostas, porém não foi constatada significância estatística nesta discordância (Tabela 2).

Tabela 2 - Distribuição do uso da USG para guiar o acesso venoso central segundo a formação dos entrevistados, em hospitais terciários da cidade de Manaus-AM, novembro de 2017 a agosto de 2018 ( $n=200)$.

\begin{tabular}{|c|c|c|c|c|c|c|c|c|c|}
\hline \multirow{4}{*}{ Variáveis } & \multicolumn{6}{|c|}{ Formação } & \multirow{4}{*}{ Total } & \multirow{4}{*}{$\%$} & \multirow{4}{*}{$\mathbf{p}^{*}$} \\
\hline & \multirow{2}{*}{\multicolumn{2}{|c|}{$\begin{array}{l}\text { Especialista } \\
n=92 ; 46 \%\end{array}$}} & \multirow{2}{*}{\multicolumn{2}{|c|}{$\begin{array}{c}\text { Residente } \\
\text { n = 93; } \\
46,5 \%\end{array}$}} & \multicolumn{2}{|c|}{$\begin{array}{c}\text { Não } \\
\text { Residente/ } \\
\text { Especialista }\end{array}$} & & & \\
\hline & & & & & \multicolumn{2}{|c|}{$n=15 ; 7,5 \%$} & & & \\
\hline & $\mathbf{f}_{\mathrm{i}}$ & $\%$ & $\mathbf{f}_{\mathrm{i}}$ & $\%$ & $\mathbf{f}_{\mathrm{i}}$ & $\%$ & & & \\
\hline \multicolumn{10}{|c|}{$\begin{array}{l}\text { Já utilizou USG para guiar a } \\
\text { punção venosa }\end{array}$} \\
\hline Sim & 69 & 75 & 68 & 73,1 & 11 & 73,3 & 148 & 74 & \multirow{2}{*}{0,956} \\
\hline Não & 23 & 25 & 25 & 26,9 & 4 & 26,7 & 52 & 26 & \\
\hline \multicolumn{10}{|l|}{ Responderam Sim } \\
\hline \multicolumn{10}{|l|}{ Frequência de uso } \\
\hline Quase sempre & 30 & 43,5 & 27 & 39,7 & 8 & 72,7 & 65 & 43,9 & \multirow{3}{*}{0,122} \\
\hline Quase nunca & 39 & 56,5 & 41 & 60,3 & 3 & 27,3 & 83 & 56,1 & \\
\hline Total & 69 & 100,0 & 68 & 100,0 & 11 & 100,0 & 148 & 100,0 & \\
\hline \multicolumn{10}{|l|}{ Motivos para não usar USG } \\
\hline Indisponível imediatamente & 45 & 65,2 & 46 & 67,6 & 10 & 90,9 & 101 & 68,2 & 0,233 \\
\hline Não existe no trabalho & 20 & 29,0 & 16 & 23,5 & 4 & 36,4 & 40 & 27,0 & 0,594 \\
\hline É mais demorado & 10 & 14,5 & 15 & 22,1 & 2 & 18,2 & 27 & 18,2 & 0,518 \\
\hline Falta de treinamento & 12 & 17,4 & 21 & 30,9 & - & - & 33 & 22,3 & $\star *$ \\
\hline Custo maior & 2 & 2,9 & 1 & 1,5 & - & - & 3 & 2,0 & ** \\
\hline
\end{tabular}

Legenda: fi: frequência absoluta simples; USG: Ultrassonografia; *Teste qui-quadrado de Pearson; **Não foi possível aplicar a estatística de teste de Pearson e de Fisher devido as restrições de Cochrane.

Fonte: Monteiro Neto AD, et al., 2021.

Dos 52 médicos entrevistados que nunca usaram a USG para guiar seus acessos venosos centrais, o principal motivo alegado foi a falta de treinamento $(69,2 \%)$, seguido da indisponibilidade imediata do aparelho $(42,3 \%)$ e da ausência do mesmo na unidade $(17,3 \%)$.

Em relação aos setores hospitalares de atuação, observou-se uma predominância na Enfermaria com 41 (20,5\%) participantes, seguido de Emergência e Centro cirúrgico com 38 (19\%) e da UTI e Emergência com 33 (16,5\%). Somente UTI, Centro Cirúrgico ou Emergência contaram com 31 (15,5\%), 31 (15,5\%) e 20 (10\%) participantes, respectivamente. $O$ menos frequente foi o grupo de profissionais que trabalhavam concomitantemente no Centro Cirúrgico e UTI, com seis (3\%) integrantes.

A frequência de punção dos entrevistados ao longo de 12 meses foi avaliada e a maioria foi composta por praticantes ocasionais, com $143(71,5 \%)$ dos entrevistados realizando menos de 25 punções por ano. As 
principais indicações clínicas que levaram os médicos a optarem preferencialmente pela punção guiada pelo ultrassom, identificadas por uma questão de múltipla escolha, foram as dificuldades anatômicas citadas por 160 (80\%) participantes, obesidade por 140 (70\%), distúrbios de coagulação por 134 (67\%) e sítio de punção da veia jugular por 64 (32\%).

O sítio preferencial para a punção foi a veia subclávia, seguido pela jugular e em último, a femoral, opção escolhida por 101 (50,5\%) participantes. Em segundo lugar, tendo sido selecionada por 79 (39,5\%) integrantes, ficou a sequência jugular, subclávia e femoral.

A indisponibilidade do ultrassom em atendimento de urgências foi referida como o principal motivo para o uso irregular entre aqueles que já possuíam alguma experiência com o aparelho. Entre os médicos participantes com experiência em punção, a maioria $99(66,9 \%)$ relatou diferentes graus de dificuldade para o acesso ao aparelho e 49 (33,1\%) relataram ter acesso ao aparelho de USG quando necessário. A maioria dos médicos 117 (58,5\%) informou que durante a graduação ou residência só lhes foi ensinada a técnica por referências anatômicas.

Aqueles que receberam treinamento em ambas as técnicas representaram $31 \%$ (62) dos participantes e os que receberam ensinamentos somente no método de acesso guiado por ultrassom foram $5 \%(10)$. 0 restante dos médicos, 11 (5,5\%), referiu que não foram educados em nenhuma das duas técnicas. A quase totalidade dos entrevistados, $196(98 \%)$, considera relevante o treinamento dos residentes na técnica tradicional anatômica.

Foi verificada uma menor tendência do uso da USG por médicos com idade igual ou superior a 50 anos, porém sem significância estatística $(p=0,062)$. A estruturação por intervalo de idades demonstra que os participantes estavam em período de vida ativa do ponto de vista profissional, a maioria deles se encontrando abaixo dos 50 anos (Tabela 3).

Tabela 3 - Distribuição etária dos médicos amostrados conforme o uso da USG como guia para o acesso venoso central, em hospitais terciários da cidade de Manaus-AM, novembro de 2017 a agosto de 2018 $(n=200)$.

\begin{tabular}{|c|c|c|c|c|c|c|c|c|}
\hline \multirow{3}{*}{ Variáveis } & \multicolumn{6}{|c|}{ Idade } & \multirow{3}{*}{ Total } & \multirow{3}{*}{$\mathbf{p}^{*}$} \\
\hline & \multicolumn{2}{|c|}{$\begin{array}{c}<30 \text { anos } \\
(n=70)\end{array}$} & \multicolumn{2}{|c|}{$\begin{array}{c}30 \mid-50 \text { anos } \\
(n=114)\end{array}$} & \multicolumn{2}{|c|}{$\begin{array}{c}\geq 50 \text { anos } \\
(n=16)\end{array}$} & & \\
\hline & $f_{i}$ & $\%$ & $f_{i}$ & $\%$ & $f_{i}$ & $\%$ & & \\
\hline \multicolumn{9}{|c|}{$\begin{array}{l}\text { Já utilizou USG para guiar a punção } \\
\text { venosa central }\end{array}$} \\
\hline Sim & 55 & 78,6 & 85 & 74,6 & 8 & 50,0 & 148 & \multirow{2}{*}{0,062} \\
\hline Não & 15 & 21,4 & 29 & 25,4 & 8 & 50,0 & 52 & \\
\hline \multicolumn{9}{|c|}{ Responderam Sim } \\
\hline \multicolumn{9}{|c|}{ Frequência de uso } \\
\hline Quase sempre & 21 & 38,2 & 42 & 49,4 & 2 & 25,0 & 65 & \multirow{3}{*}{0,230} \\
\hline Quase nunca & 34 & 61,8 & 43 & 50,6 & 6 & 75,0 & 83 & \\
\hline Total & 55 & 100,0 & 85 & 100,0 & 8 & 100,0 & 148 & \\
\hline
\end{tabular}

Fonte: Monteiro Neto AD, et al., 2021.

\section{DISCUSSÃO}

Neste estudo, verificamos que dos 200 profissionais questionados, 148 (74\%) já haviam feito uso da USG como guia na inserção de cateteres venosos centrais. Destes, $65(43,9 \%)$ referiram usar rotineiramente a tecnologia, taxa inferior às obtidas em outros países, 55-68\% (MAIZEL J, et al., 2016; MATAVA C e HAYES J, 2011; SCHOLTEN HJ, et al., 2019). A heterogeneidade da amostra composta por anestesistas, clínicos, emergencistas e intensivistas pode ter influenciado nessa menor frequência, visto que algumas dessas especialidades tem maior tendência à utilização rotineira do ultrassom, tais como os anestesistas e os intensivistas e outras, menor tendência, como os clínicos. Em outros estudos, a maior taxa de utilização pode ser relacionada a amostra ser composta por uma só especialidade, intensivista ou anestesista (MAIZEL J, et al., 2016; MATAVA C e HAYES J, 2011). 
Zieleskiewicz L, et al. (2015) mostraram que a USG para guiar punções venosas centrais foi utilizada apenas em pouco mais que a metade (54\%) das indicações em Unidades de Tratamento Intensivo (UTI) da França, Bélgica e Suíça. Já entre intensivistas franceses, Maizel J, et al. (2016) obtiveram uma taxa de uso de $68 \%$, no entanto, toda a comunidade estudada havia recebido anteriormente treinamento na técnica e havia aparelhos de ultrassom disponíveis em todas as UTIs. Scholten HJ, et al. (2019) encontraram taxa equivalente entre os anestesistas e intensivistas holandeses.

Segundo Matava C e Hayes J (2011), os participantes que exerciam seu trabalho em hospitais acadêmicos do Canadá possuíam frequência de uso da USG de $60 \%$, enquanto aqueles que trabalhavam em unidades hospitalares exclusivamente comunitárias e assistenciais tinham taxa inferior (33\%). Na amostra estudada não conseguimos realizar esta comparação, visto que em nossa realidade os profissionais trabalham nos setores públicos e privados simultaneamente. Somente $13(8,8 \%)$ participantes que faziam uso da punção guiada por USG trabalhavam apenas no setor privado.

A questão socioeconômica do Brasil também pode justificar a diferença dos resultados encontrados em países europeus e norte-americanos (MATAVA C e HAYES J, 2011; ZIELESKIEWICZ L, et al., 2015). Entretanto, vale ressaltar que todos os hospitais deste estudo possuíam aparelhos de USG, ainda que não imediatamente disponíveis. Há de se considerar a possibilidade do pouco uso ser justificado pela falta de treinamento o que levaria à preferência da punção de veia subclávia por meio da técnica anatômica, observado em 101 (50,5\%) participantes deste trabalho. Pellegrini JAS, et al. (2018) encontram, no Brasil, esta preferência entre os participantes sem treinamento $(48,4 \%)$, em contraste com a predileção pela punção da veia jugular interna assistida por USG entre aqueles treinados (41\%).

Entre os médicos que já haviam realizado ao menos uma punção venosa guiada por USG, o fator preponderante foi a indisponibilidade imediata do aparelho $(68,2 \%)$. Entre os que jamais fizeram uso da tecnologia, a barreira mais citada foi a falta de treinamento $(69,2 \%)$. Em outros estudos, os principais motivos pelos quais os médicos não usaram a técnica foram disponibilidade limitada do aparelho, aumento do tempo de procedimento e preocupação com a perda de habilidades na técnica anatômica (SCHOLTEN HJ, et al., 2019; SONI NJ, et al., 2016; ZAVER F, et al., 2018).

A indisponibilidade do aparelho de USG em situações de emergência foi identificada por 99 (66,9\%) médicos com experiência em punção, valor bem superior ao observado em um estudo holandês, no qual foi verificada uma frequência de apenas 17,2\% (SCHOLTEN HJ, et al., 2019). Neste estudo, a ausência de benefícios que justifiquem o uso do ultrassom foi relatada por 24 (12\%) médicos, índices inferiores aos encontrados em estudos prévios (20,9-59,8\%) (MAIZEL J, et al., 2016; MATAVA C e HAYES J, 2011; SCHOLTEN HJ, et al., 2019). Entre os participantes parece já haver o entendimento de que a utilização da técnica guiada por USG se traduz em maior segurança para os pacientes, faltando resolver apenas 0 problema da ausência de treinamento e da indisponibilidade de equipamentos.

Sobre a técnica anatômica, 196 (98\%) participantes acreditam que ela ainda deve ser ensinada aos residentes, visto a possibilidade de falta de aparelhos e da escassez de treinamento. Entretanto, esse percentual tende a ser menor quando o aparelho de USG é implementado de forma regular nas unidades, onde o único motivo para se usar a orientação por planos anatômicos seria a chamada "punção de emergência" em que não seria possível esperar a chegada do equipamento (MAIZEL J, et al., 2016; MATAVA C e HAYES J, 2011; BAILEY PL, et al., 2007). Em relação aos residentes e especialistas, não houve diferenças com significância estatística entre os dois conjuntos. Para os residentes, a ausência de treinamento representou uma barreira mais relevante do que para o grupo dos especialistas, 31\% e 17\%, respectivamente. Maizel J, et al. (2016) encontraram índices inferiores na França, com o grupo de residentes atribuindo em cinco por cento a ausência de treinamento, ao passo que o grupo de especialistas relatou $14 \%$.

Houve uma preferência neste estudo pela punção da subclávia (50,5\%), local menos propenso ao uso da USG pelas barreiras ósseas e, portanto, mais abordado usando a técnica anatômica tradicional. Relevante ressaltar que o método de acesso da veia subclávia ou femoral com a utilização do ultrassom como guia é possível e possui equivalência ao método de utilização de planos anatômicos do ponto de vista de segurança e eficácia (BRASS P, et al., 2015). Os limites deste estudo foram a multiplicidade de especialidades 
envolvidas, o número de hospitais analisados e a padronização do tipo de aparelho disponível para a punção guiada pela USG. Outros estudos deverão ser realizados contemplando estas variáveis para a melhor compreensão dos aspectos que levam ao baixo índice de utilização do aparelho de USG nas punções venosas.

\section{CONCLUSÃO}

Após análise dos dados coletados nesse estudo, concluímos que há um baixo índice de utilização da ultrassonografia para acesso venoso central na população estudada. Visto os vários benefícios encontrados na literatura a respeito da utilização deste método para a punção venosa central, considera-se que algumas ações devem ser realizadas, tais como, maior disponibilidade de aparelhos de ultrassom nas unidades assim como um programa de educação continuada para estimular a adoção da tecnologia pelos profissionais.

\section{AGRADECIMENTOS E FINANCIAMENTO}

À Fundação de Amparo à Pesquisa do estado do Amazonas (FAPEAM) que por meio do Programa de Apoio à Publicações Científicas (PAPAC), edital 005/2019 permitiu a realização deste estudo.

\section{REFERÊNCIAS}

1. BAILEY PL, et al. A survey of the use of ultrasound during central venous catheterization. Anesthesia \& Analgesia, 2007; 104(3): 491-497.

2. BANNON MP, et al. Anatomic considerations for central venous cannulation. Risk management and healthcare policy, 2011; 4: 27-39.

3. BRASS $P$, et al. Ultrasound guidance versus anatomical landmarks for internal jugular vein catheterization. Cochrane Database of Systematic Reviews, 2015(1).

4. FRANCO-SADUD R, et al. Recommendations on the Use of Ultrasound Guidance for Central and Peripheral Vascular Access in Adults: A Position Statement of the Society of Hospital Medicine, Journal of Hospital Medicine, 2019; 14: E1-E22.

5. GREBENIK CR, et al. NICE guidelines for central venous catheterization in children. Is the evidence base sufficient? British Journal of Anaesthesia, 2004; 92(6): 827-830.

6. KASATKIN AA, et al. Ultrasound-guided internal jugular vein cannulation in the obese patient in a forced semi-sitting position. The journal of vascular access, 2018; 19(4): 398-400.

7. LAMPERTI M, et al. International evidence-based recommendations on ultrasound-guided vascular access. Intensive Care Medicine, 2012; 38(7): 1105-17.

8. LAU CS, CHAMBERLAIN RS. Ultrasound-guided central venous catheter placement increases success rates in pediatric patients: a meta-analysis. Pediatric Research, 2016; 80(2): 178-184.

9. LEGLER D, NUGENT M. Doppler localization of the internal jugular vein facilitates central venous cannulation. Anesthesiology, 1984; 60(5): 481-482

10. MAIZEL J, et al. Practice of ultrasound-guided central venous catheter technique by the French intensivists: a survey from the BoReal study group. Annals of Intensive Care, 2016; 6(1): 76.

11. MATAVA C, HAYES J. A survey of ultrasound use by academic and community anesthesiologists in Ontario. Canadian Journal of Anesthesia/Journal canadien d'anesthésie, 2011; 58(10): 929-935.

12. MONTEIRO NETO AD. Avaliação da prática de punção venosa central guiada por ultrassom em Manaus/AM. Dissertação (Mestrado Profissional em Cirurgia) - Programa de Pós-Graduação em Cirurgia. Universidade Federal do Amazonas, Manaus, 2019; $67 \mathrm{p}$.

13. NATIONAL INSTITUTE FOR CLINICAL EXCELLENCE. NICE technology appraisal guidance No 49: guidance on the use of ultrasound locating devices for placing central venous catheters. London: NICE. 2002.

14. NORITOMI DT et al. Evaluation of cost-effectiveness from the funding body's point of view of ultrasound-guided central venous catheter insertion compared with the conventional technique. Revista Brasileira de Terapia Intensiva, 2016; 28(1): 62 .

15. OULEGO-ERROZ I, et al. Ultrasound-guided or landmark techniques for central venous catheter placement in critically ill children. Intensive Care Medicine, 2018; 44(1): 61-72.

16. PELLEGRINI JAS, et al. Point-of-care ultrasonography in Brazilian intensive care units: a national survey. Annals of Intensive Care, 2018; 8(1): 50.

17. PETERS JL, et al. Doppler ultrasound technique for safer percutaneous catheterization of the infraclavicular subclavian vein. American journal of surgery, 1982; 143(3): 391-3.

18. SAUGEL B, et al. Ultrasound-guided central venous catheter placement: a structured review and recommendations for clinical practice. Critical Care, 2017; 21(1): 225.

19. SCHOLTEN HJ, et al. Barriers to ultrasound guidance for central venous access: a survey among Dutch intensivists and anaesthesiologists. Journal of clinical monitoring and computing, 2019; 33(6): 1023-31. 
20. SONI NJ, et al. Use of ultrasound guidance for central venous catheterization: a national survey of intensivists and hospitalists. Journal of Critical Care, 2016; 36: 277-283.

21. TROIANOS CA, et al. Guidelines for performing ultrasound guided vascular cannulation: recommendations of the American Society of Echocardiography and the Society of Cardiovascular Anesthesiologists. Journal of the American Society of Echocardiography, 2011; 24(12): 1291-1318.

22. YONEI A, et al. Real-time ultrasonic guidance for percutaneous puncture of the internal jugular vein. The Journal of the American Society of Anesthesiologists, 1986; 64(6): 830-831.

23. YOUNG MP, YOU TH. Complications of central venous catheters and their prevention. Up to Date, Topic 8180 , version 15.0., 2020. Literature review current through Nov, 2020

24. ZAVER F, et al. International Scope of Emergency Ultrasound: Barriers in Applying Ultrasound to Guide Central Line Placement by Providers in Nairobi, Kenya. Emergency medicine international, 2018; 2018.

25. ZIELESKIEWICZ L, et al. Point-of-care ultrasound in intensive care units: assessment of 1073 procedures in a multicentric, prospective, observational study. Intensive Care Medicine, 2015; 41(9): 1638-47. 